\title{
Design and Implementation of an Electric-driven Self-propelled Handling Equipment
}

\author{
Chi Ruijuan ${ }^{1 *}$, a Hu Tao ${ }^{1, b}$, Li Han ${ }^{1, c}$, Du Yuefeng ${ }^{1, d}$, Zhang Zhen ${ }^{1, \mathrm{e}}$, Xie Bin $^{1, \mathrm{f}}$ \\ ${ }^{1}$ College of Engineering, China Agricultural University, Beijing 100083, China \\ achiruijuan@cau.edu.cn, b513947768@qq.com, coracelihan@163.com, ddyf@cau.edu.cn, \\ 535336503@qq.com, 'xb0306@cau.edu.cn
}

Keywords: Electric-driven self-propelled, Bagged agricultural materials transportation, Train carriage, Handling equipment, Control system.

\begin{abstract}
In view of the present situation that the automation level of handling equipment for bagged agricultural materials in railway transportation is low in China, a specific electric-driven self-propelled handling equipment was designed, which can move automatically in the narrow train carriage to transport bagged agricultural materials. The handling equipment was mainly composed of steel frame structure, and used six wheels with dual driving wheels in center, an independent driving unit with two motors. The control system was designed based on Freescale K60, it can realize the straight moving and the in-situ steering through the infrared remote control. Then the handling equipment was manufactured and tested. The experimental results showed that the designed electric-driven self-propelled handling equipment could run reliably by the infrared remote control, and it has preferable load performance and possibility. It can realize the in-situ steering with little space, meeting the requirements of transporting bagged agricultural materials in narrow train carriage. The design can provide reference for the modernization of agricultural material logistics equipment, and provide a preliminary experimental platform for further study of the automatic control of agricultural materials handling equipment.
\end{abstract}

\section{Introduction}

China is a large agricultural country. At present, the majority of food, fertilizers, feed and other powdered agricultural products are transported in woven sacks by the train. Most bags are loaded and unloaded manually in China. These bagged agricultural materials are heavy in weight, and some of them are chemical corrosive and toxic. Moreover, the whole work environment is basically filled with dust and noise. Working in such an environment is not only labor intensive and inefficient, but also will cause irreversible damage to both the physical and mental health of the workers. In addition, according to the field research, the current age of the staff was generally large and there was a subsequent shortage of human resources, which is an urgent problem such a logistics enterprise need to solve.

In recent years, AGV [1-6] has been widely used at home and abroad. But most of them still apply to large space such as factories and warehouses, which are not easy to be used in small space. At the same time, automatic navigation way of AGV [7,8] such as electromagnetic guidance, optical guidance, tape guidance, and laser guidance, needed to fix the facilities in the workplace, which is difficult to realize in the train logistics with highly mobility. Aim at the above problems, a large electric-driven self-propelled handling equipment for the train logistics was designed in this paper in order to realize the mechanization and automation of the agricultural transportation operations in the railway carriage.

\section{Structural design of the handling equipment}

The electric-drive self-propelled handling equipment designed in this paper will carry on the transportation of agricultural materials with pallets in the train carriage. In order to adapt to the 
narrow space in the train carriage, it required not only the handling equipment to be small enough while meeting strength and stiffness, but also the turning radius as small as possible. Therefore, according to the size of the train carriage and the tray, the designed handling equipment was $1000 \mathrm{~mm}$ long, $850 \mathrm{~mm}$ wide, the total height of it is $731 \mathrm{~mm}$, and the height of the chassis is $281 \mathrm{~mm}$. In order to reduce the steering radius, the handling equipment used a six wheel dual drive system, and the two drive wheel was located in the middle of the vehicle [9]. The power source were two $48 \mathrm{~V}-1.5 \mathrm{KW}$ brushless DC motors. At the same time, a battery pack connected by four $12 \mathrm{~V}$ lead acid batteries was used as the power supply, and the battery type is $6 \mathrm{D} 155$.

The six wheel dual driving wheel center structure not only made the equipment balance and bearing capacity better, but also the steering of the handling equipment more flexible. Moreover, through the differential rotation of the two driving wheels, the in-situ steering function can be realized [10-12]. The utility model was characterized in that the drive unit was relatively independent, and the tray support, the chassis and the universal wheel were welded as a whole support frame. And the driving unit was connected with the whole support frame through springs, steel plates and bolts. The overall structure design of handling equipment is shown in figure 1. The drive unit mainly included drive wheel, drive unit, support frame, drive motor, flange, drive shaft, deep groove ball bearing and bearing seat, as shown in figure 2 .

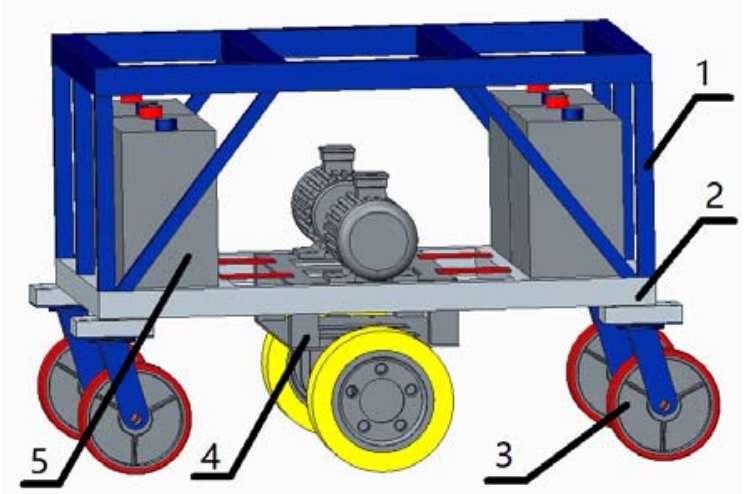

1.Pallet holder 2.Chassis 3. Universal wheel 4. Driving unit 5 Battery

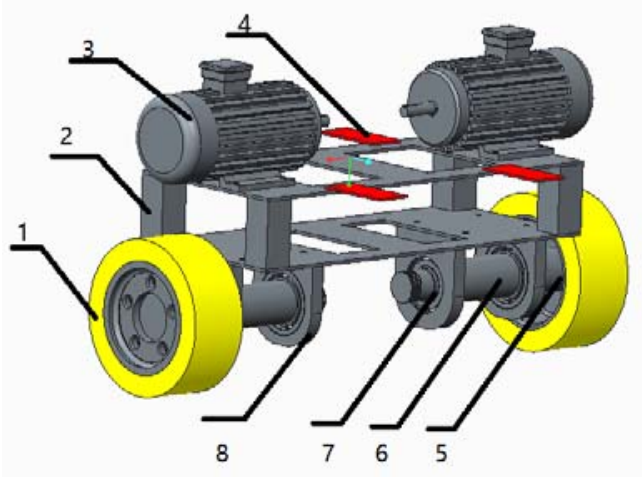

1. Driving wheel 2.The carriage of driving unit 3. Motor 4. Board springs 5. Flange 6. Drive shaft

7. Deep groove ball bearing 8 . Bearing seat

Fig.2 Driving unit

Fig.1 The overall structure of handling equipment

The spring steel plate in Figure 2 not only had the function of connecting, but also made the handling equipment have a certain elasticity and shock absorption ability. It can ensure that the equipment can reach the ground in six wheels in most working conditions, and the force distribution is uniform. The motor and the drive shaft were driven by gears and chains.

\section{Design of the control system of the handling equipment}

\subsection{General scheme of control system}

The electric-drive self-propelled handling equipment adopted Freescale Carle K60 [13] series chip as the control core, and was controlled by infrared remote control. When walking, two speed signals of the driving wheel were collected by photoelectric encoder, and feedback control was carried out to ensure the straight walk of the device. At the same time, ultrasonic ranging sensor was used as auxiliary to realize obstacle stopping during walking. The composition of the control system is shown in figure 3. 


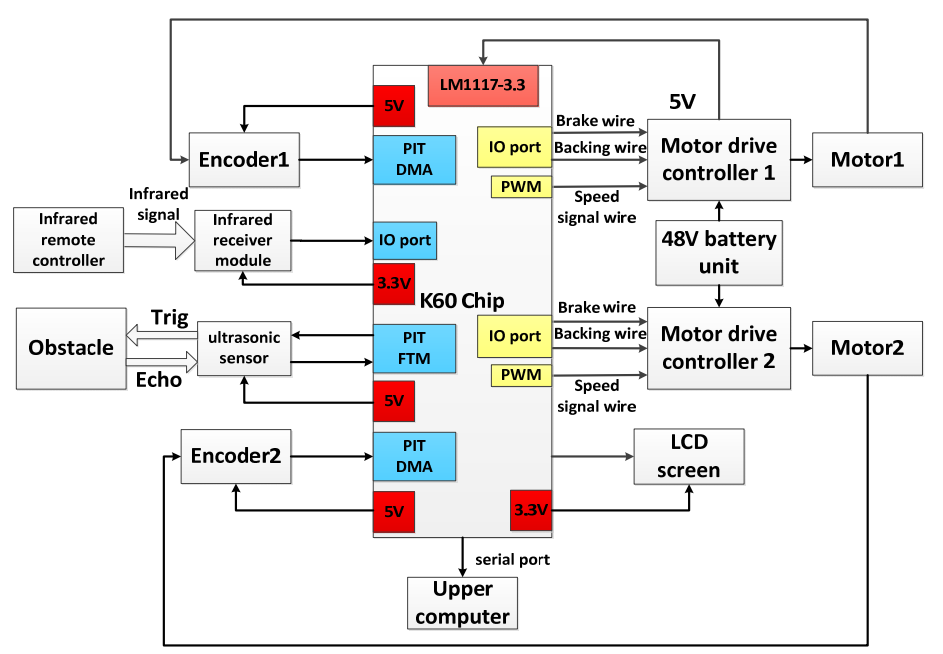

Fig.3 Controller system

The infrared receiving module of the control system adopted the VS1838 type. The photoelectric encoder selected has a resolution of $600 \mathrm{P} / \mathrm{R}$. The ultrasonic ranging sensor used the HC-SR04 type. The size of the LCD display screen is 3.2 inches, and the resolution is $320 * 240$. According to the selected device, the hardware circuit of the whole control system was designed, as shown in figure 4 . According to the schematic diagram of the circuit, the control circuit board was made as the hardware foundation of the handling equipment.

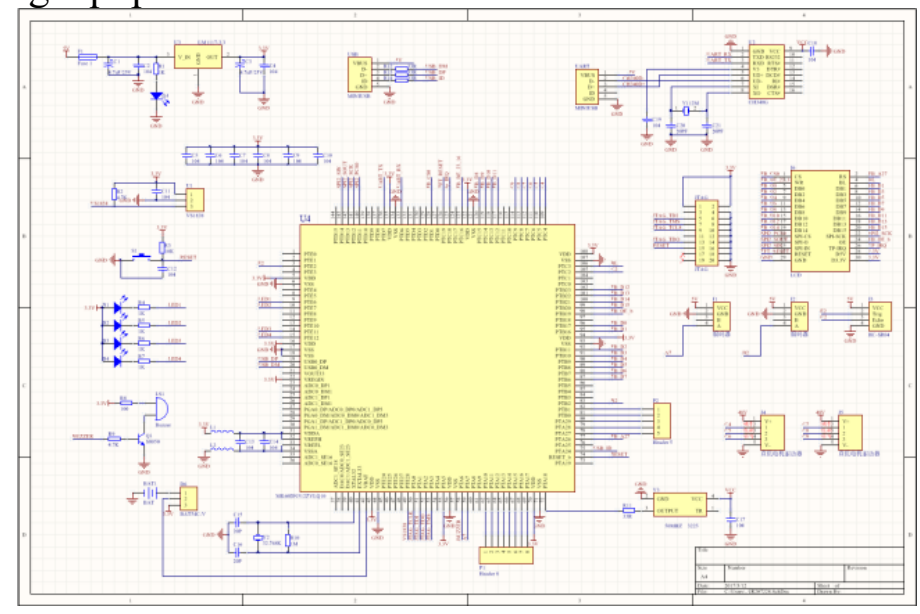

Fig.4 circuit schematic

\subsection{Control system software design}

\subsubsection{General strategy of control system}

The overall control strategy of the control system is shown in figure 5. After the system was powered on, the initialization was completed. Then, whether the handling equipment can move was determined according to the information measured by the ultrasonic distance measuring sensor. Finally, the control chip controlled the working state of the two motors according to the different key information received by the infrared receiving module, to realize the actions of forward, backward, in-situ, left and right pivot steering and stop. At the same time, the encoder transmitted the collected speed information to the control chip, and adjusts the speed of the two drive wheel according to analysis and comparison of signals from the speed sensors. The display function is not shown as an auxiliary function in the overall flowchart. 


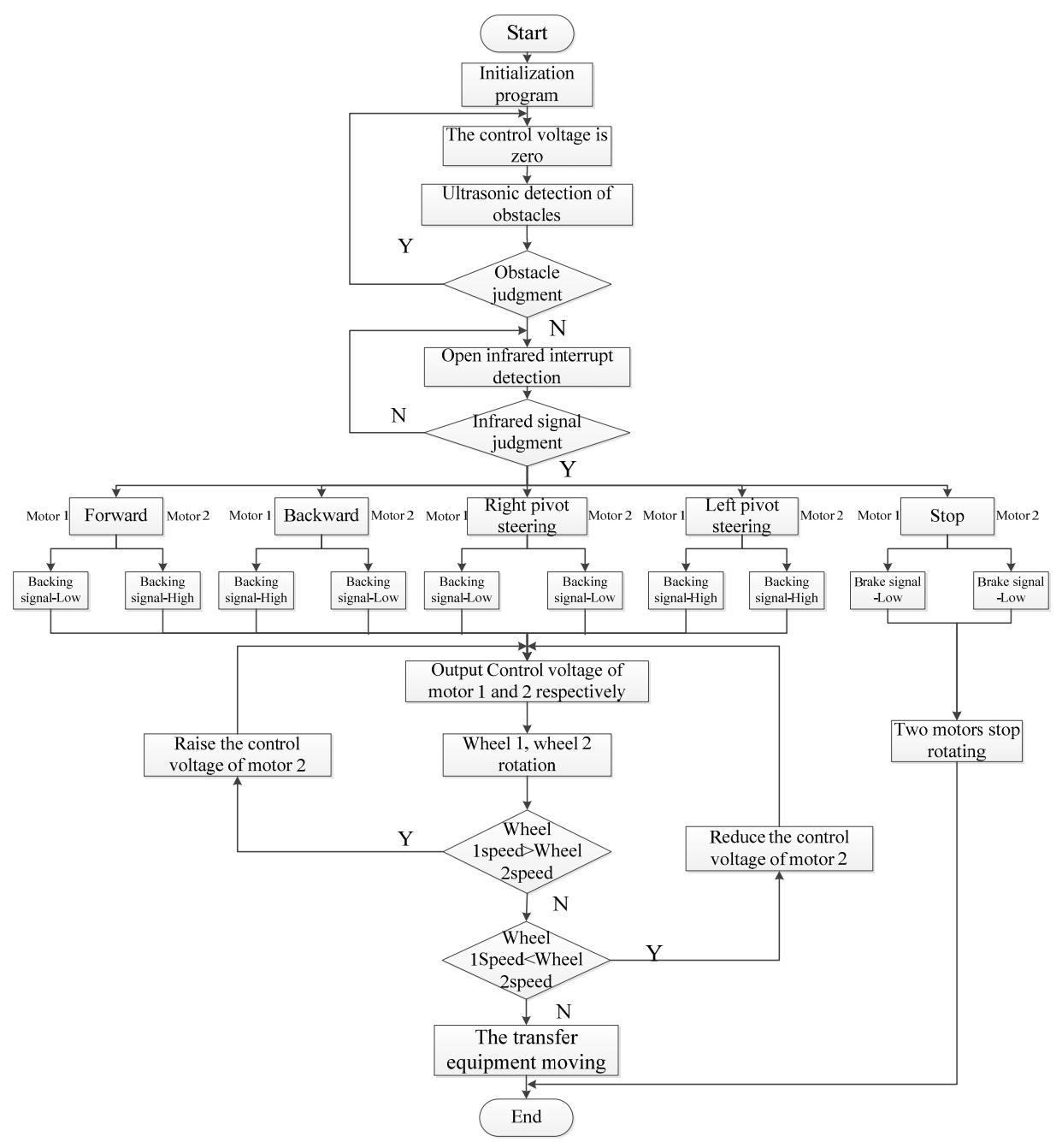

Fig.5 Integrated control strategy

\subsubsection{Motor control}

The driving motor of the handling equipment used was the permanent magnet brushless DC motor $[14,15]$. The drive motor has a complete set of motor drive controller. There were three control wires on the motor drive controller, the low level brake wire, the backing wire and the speed signal wire. Low level brake wire and backing wire were active for low level signal. While the speed signal wire receives analog voltage signal, and the range is $0 \sim 3.3 \mathrm{~V}$. Different voltage corresponding to different speed, the higher the voltage, the faster the speed. Therefore, only control of high and low level signal of the control pin on the control board was required for controlling of the low level brake wire and the backing wire. As for the speed control, it was necessary to produce a PWM wave with a certain duty cycle on the control pin to form the corresponding analog voltage.

\subsubsection{Infrared control}

The handling equipment was controlled by infrared remote control, so it was necessary to decode and judge the infrared signal issued by infrared remote controller. The infrared program was based on NEC standard infrared decoding [16, 17]. The control chip can identify different keys on the infrared remote controller by judging the key code obtained by decoding, so that the corresponding control can be performed.

\subsubsection{Encoder information acquisition and feedback}

The detection of the rotation speed of the two driving wheel was realized by an optical encoder [18], and the encoder shaft is connected with the shaft center of the drive shaft through a flexible coupling. By comparing the speed of the two driving wheel by the control chip, the handling equipment can walk straightly. Specific operations were as follows: the rotation speed of one of the two motors (denoted as motor 1) was used as a reference. When the rotation speed of another motor 
(denoted as motor 2) was lower than the motor 1, the control chip raised a certain voltage by appropriately increasing the duty ratio of the control pin of the motor 2 so as to appropriately increase the speed of the motor. When the motor 2 speed was higher than the motor 1, then vice versa. By this method, the speed of the two motors was basically the same, so that the deviation of the device during the straight walk was within the allowable range.

Secondly, in order to the using encoder to realize automatic control of handling equipment about 90 degrees left and right pivot steering, the strategy adopted was to detect and control the number of turns of the driving wheel when steering. When the steering wheel conducted a 90 degrees pivot steering, the running path of the two driving wheels on the ground was a circle, and each driving wheel passes through 1/4 circles. According to the quantitative relationship between the length of the trajectory and the circumference of the drive wheel, the moving device was rotated about 0.73 circle when it was conducting a 90 degrees pivot steering, and the coaxial encoder shaft was rotated 0.73 circle accordingly. The number of pulses emitted by the encoder in each turn is 600 , and the number of pulses corresponding to the 0.73 turns is 438 . The number of pulses is counted by the interruption of the pin, and when the value reaches 438 , the control chip controls the stopping of the two motor and realizes the automatic 90 degrees left or right pivot steering.

\subsubsection{Obstacle distance detection}

The working principle of ultrasonic sensor obstacle detection is that the IO port is used to output the high level signal at a certain time on the Trig port of the sensor to trigger ranging. The module automatically sent eight $40 \mathrm{KHz}$ square waves and automatically detected if there was a signal to return. When a signal was returned, the Echo port of the sensor outputted a high level to the corresponding IO port. The duration of the high level is the ultrasonic distance from the launch to the return, and the detection distance $=($ high level time $*$ sound speed) $/ 2$. Through the distance signal processing, whether the obstacles in the safe range was determined. The safe distance was $30 \mathrm{~cm}$.

\section{Experiments}

The electric-driven self-propelled handling equipment has been trial-manufactured, shown in Fig.6. To verify that each part of the handling equipment meets the design requirements and the reliability of the control system, experiments on straight-line walking, pivot steering and handling on load were carried out indoor. The speed of the handling equipment was controlled within $0.5 \mathrm{~m} / \mathrm{s}$ during the experiments.

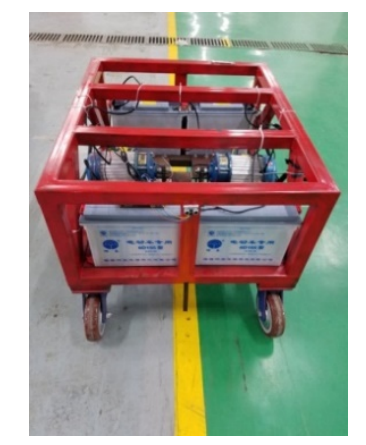

Fig.6 The electric-driven self-propelled handling equipment

\subsection{Straight-line walking experiment}

Straight-line walking experiment was carried to verify the control precision of the handling equipment when walking in straight line. An eight-meter route was chosen, the starting and terminal position were marked by white slips. The handling equipment was walking beyond a yellow line of $109 \mathrm{~mm}$ in width, which was used as a reference to judge the deviation of line walking. The design goal is that the deviation is no more than the width of the yellow line after walking eight meters in straight line, that is, the deviation rate is less than 1.4\%. Fig.7 shows the straight-line walking process, including the starting, middle and terminal position. 


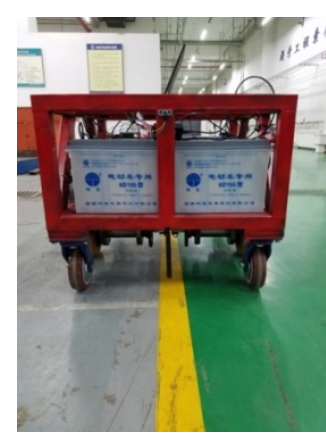

(a) Starting position

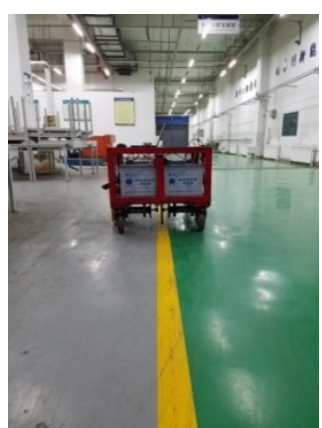

(b) Middle position

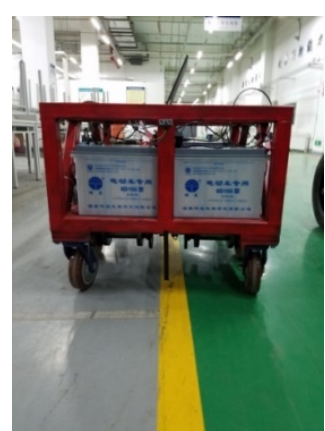

(c) Terminal position

Fig.7 Straight-line walking process (Photos)

During the low speed walking of the handling equipment, the change of the frequency (speed) of the two driving wheels was measured, as shown in Fig.8 (Intercepted a piece of data from the starting point of the handling equipment).

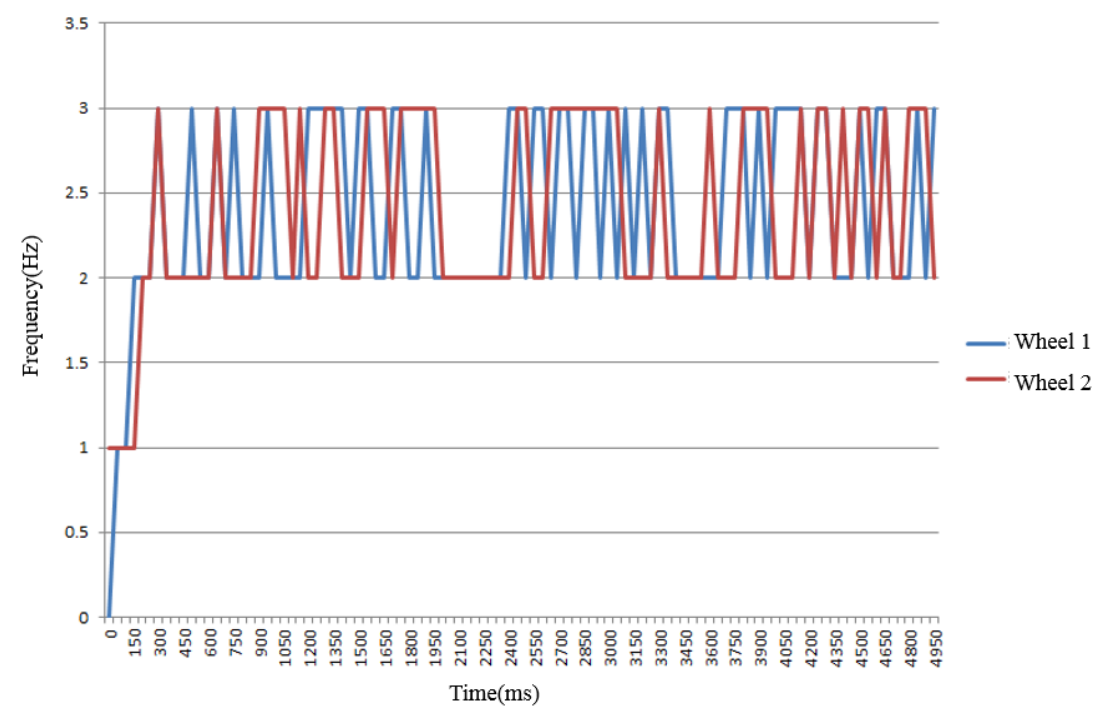

Fig.8 Speed change of driving wheels

Under the feedback control, the speed of the two driving wheels fluctuates in a small range and basically keeps stable, thus ensuring that the handling equipment can walk in a straight line.

\subsection{Pivot steering experiment}

This experiment was carried to verify the feasibility of pivot steering of the handling equipment, and observe the slip degree of the handling equipment while pivot steering. The dimensions of the handling equipment are known to be $1000 \mathrm{~mm} \times 850 \mathrm{~mm}$ (length and width). Pivot steering can be regarded as the rotation motion centered on its center, so the minimum space for pivot steering is a circle with a diameter of approximately $1312 \mathrm{~mm}$, as shown in Fig.9.

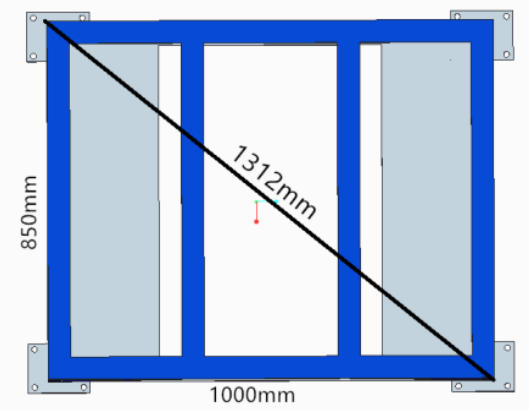

Fig.9 The minimum space for pivot steering 
A square area with a side length of $1360 \mathrm{~mm}$ was labeled as the experimental region. According to design intention, the handling equipment would be proved to have a good performance of pivot steering if it is still in the marked square area after several continuous turns in situ. Fig.10 shows the starting, intermediate and final state of the handling equipment during the experiment.

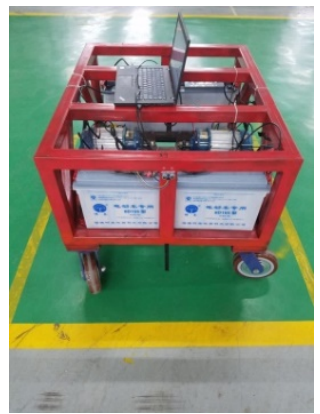

(a) Starting state

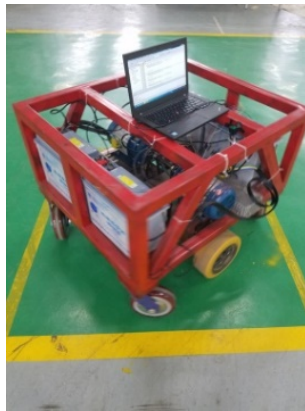

(b) Intermediate state

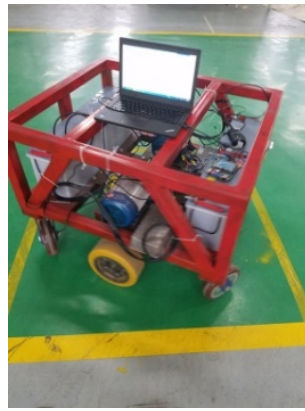

(c) Intermediate state

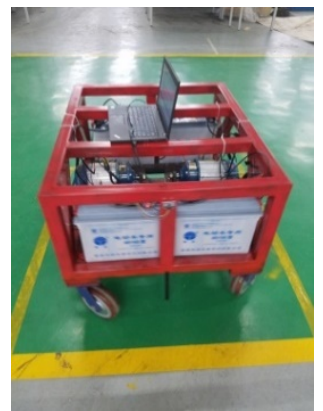

(d) Final state

Fig.10 The experimental process of pivot steering

As Fig.10 shows, the handling equipment eventually stopped in the marked square area after several continuous turns in situ, and there is not too much slip compared with the initial position. By observing, the handling equipment had not been beyond the square area during the whole experiment. It is proved that the handling equipment has a good performance of pivot steering, such as turning in situ to any angle, good stability, and small space for pivot steering.

\subsection{Handling experiment with heavy load}

On the basis of the experiment of straight walking and pivot steering, it was necessary to carry out the handling experiment with heavy load on the designed handling equipment to test the feasibility and load capacity. In the experiment, the blue tray was placed at the top of the handling equipment, and it was stacked with bagged agricultural materials. Then, the handling equipment was controlled to carry out the transportation operation, as shown in figure 11.

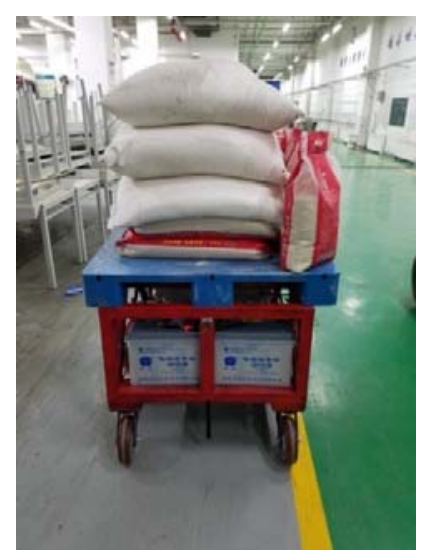

Fig.11 Experiment of carrying capacity

The tray was stacked with bags of flour $(50 \mathrm{Kg} / \mathrm{bags})$ and rice $(50 \mathrm{Kg} / \mathrm{bags})$, with a total weight of more than 1 tons. The whole transportation process was stable in power output and smooth in handling. Pallets and bags of agricultural materials had no tendency to fall. The experiment proved that the designed handling equipment has better stability and has a certain load capacity.

\section{Conclusion}

(1) According to the requirements of transporting farm bags of agricultural materials in the narrow railway carriage, the overall mechanical structure scheme of the electric drive self-propelled handling equipment was determined. 
(2) The overall scheme of electric-drive self-propelled handling equipment control system was designed. A software and hardware control system based on Freescale K60 control chip was developed, including double motor drive control, infrared remote control, walking steering control, encoder acquisition and feedback control, ultrasonic sensor ranging control.

(3) A prototype of electric drive self-propelled handling equipment was developed and related experiments were carried out. The experimental results showed that the developed electric drive self-propelled handling equipment and its control system can reliably realize the functions of straight walking, in-situ steering and load handling by infrared remote control. The handling equipment had better load performance and passability, and the steering space required for in-situ steering was small, which basically met the requirement that the handling equipment carries the operation of carrying agricultural materials in a narrow railway carriage.

(4) The designed handling equipment drive control system had stable performance, reliable data communication, modular control unit, and can realize effective control of electric-drive self-propelled handling equipment. This laid the foundation for the follow-up handling equipment running in the complex conditions, for example also provided an experimental platform for future research on more advanced control such as visual control, to make the handling equipment more intelligent.

\section{References}

[1] Zhang Chenbeixi, Huang Zhiqiu. Evolution Summarization of Automated Guided Vehicles(AGV) [J]. Manufacture Information Engineering of China, 2010，39（1）: 53-59.

[2] Li Shujun, Wang Jun, Liu Yuzhou. Analysis and Discussion of New Intelligent Logistics Equipment and Key Technologies[J]. Mechanical Engineering \& Automation, 2012, (2) : $212-213,216$.

[3] Wang Rongben, Zhang Youkun, Wang Zhizhong. Development of AGVS in the World[J]. Transactions of the Chinese Society of Agricultural Engineering, 1994, 10 (2): $18-22$.

[4] M Collier, A micro-AGV for flexible manufacturing in small enterprises[J]. Integrated Manufacturing Systems, 2003,(14):442—448.

[5] IFA Vis, Survey of research in the design and control of automated guided vehicle system[J].European Journal of Operational Research. 2006,(170):677—699.

[6] N Jawahar, ed. AGV schedule integrated with production in flexible manufacturing systems[J]. The International Journal of Advanced Manufacturing Technology 1998,14 (6) : 428-440.

[7] Yang Xianlong, Design of stucture and control for two-wheel difference spped steering AGV with magmetic navigation[D].He Fei: Hefei University of Technology,2014

[8] Niu Kun, Stucture design and path planning of automated guide cehicle[D].Beijing: Beijing University of Posts and Telecommunications,2014.

[9] Zhang Hanbin,The research of logistics transport vehicle(AGV) for E-commerce distibuton center[D]. Beijing: Beijing Wuzi University, 2014.

[10] Chu Jiangwei, Gu Baiyuan, Wang Rongben. Design on AGV with Two Wheels Different Speed steering Based on Machine Vision[J]. Logistics Technology, 2002， (7) : 12-14,19.

[11] Lu Shan, Mation contol of MCU-based differential drive automated guide vehicle [D]. Xi'an: Xi'an University of technology,2010.

[12] Gu Yuchuan. Study on the driving and steerig integration mechanism and autonomous navigation control of AGV[D].Chang Chun: Jilin University,2006.

[13] Freescale Semiconductor Inc. K60 Data Sheet[DB/OL].http://www.Freescale.com. 
[14] SP Natarajan, ed. Implementation of a new digital controller for a permanent magnet brushless DC motor[J]. Industrial Electronics,2000(1):145-150.

[15] Zhang Chen. Principle and application of Brushless DC motor [M]. Beijing: China Machine Press, 2004.

[16] Nie Shiliang, Li Leimin. A method of sending and receiving infrared remote-control signal with microchip computer[J]. Information Technology, 2004, 28 (2) : 21-23,96.

[17] Li Liang, The design of system of IR remote controlled vehicle based on atmegal 16[D]. Kubming:Kunming University of Science and Technology,2011.

[18] Ding Weidong, Zhu Weimin,Cao Lingzhi. Design of motor speed-measuring system based on the incremental photoelectric encoder[J]. Journal of Zhengzhou University of Light Industry, 2013,28 (6) : 95-97. 